\title{
Bipolar hemiarthroplasty versus total hip replacement in displaced femoral neck fracture in elderly
}

\author{
Choudhary MB. ${ }^{1}$, Ram GG. ${ }^{2 *}$
}

DOI: https://doi.org/10.17511/ijoso.2020.i02.07

\footnotetext{
${ }^{1}$ Mohan B Choudhary, Associate Professor, Department of Orthopaedics, Sri Ramachandra Medical College, Chennai, Tamil Nadu, India.

2* Ganesan G Ram, Associate Professor, Department of Orthopaedics, Velammal Medical College, Madurai, Tamil Nadu, India.
}

Introduction: Fractures of the neck of the femur are commonly seen in the geriatric population resulting in significant morbidity and mortality. Primary arthroplasty helps in rapid mobilization while decreasing the chances of implant failure and complications such as deep vein thrombosis, pulmonary complications, infection, and bedsores. The purpose of this can be fulfilled either by Bipolar Hemiarthroplasty as well as by Total Hip arthroplasty. The aim of this study is to determine whether total hip replacement is clinically and functionally better than bipolar hemiarthroplasty in the neck of femur fracture in older patients. Method: It is a prospective study done at Sri Ramachandra Medical College, Chennai, and Velammal Medical College, Madurai on 70 patients diagnosed with the unstable neck of femur fractures who were treated with bipolar hemiarthroplasty and total hip replacement from April 2018 to September 2019. The inclusion criteria were age more than 60 years and Unstable neck of femur fractures as per the Garden classification. The exclusion criteria were pathological fractures, associated fracture of lower limbs, and non-ambulatory patients prior to the injury. A functional assessment was carried out using the Harris Hip Score. Results: In the group that underwent a total hip replacement, the mean Harris Hip Score was 70.6, 74.4, 77.68 and 80.95 at the follow-up visits at week 6,3 months, 6 months and 1 year respectively. In the group that underwent bipolar hemiarthroplasty, the mean Harris Hip Scores were 63.50, 66.25, 68.72 , and 71.26 at the follow-up visits at week 6,3 months, 6 months and 1 year respectively. Conclusion: Total hip arthroplasty is recommended over hemiarthroplasty for the displaced neck of femur fractures in elderly patients owing to the higher Harris Hip Scores and lower re-operation rates.

Keywords: Neck of the femur, Bipolar Hemiarthroplasty, Harris Hip score, Osteoporosis, Total Hip replacement

\section{Corresponding Author}

Ganesan G Ram, Associate Professor, Department of Orthopaedics, Velammal Medical College, Madurai, Tamil Nadu, India.

Email: ganesangram@yahoo.com

\section{How to Cite this Article}

Choudhary MB, Ram GG. Bipolar hemiarthroplasty versus total hip replacement in displaced femoral neck fracture in elderly. Surgical Review Int J Surg Trauma Orthoped. 2020;6(2):105-109.

Available From

https://surgical.medresearch.in/index.php/ijoso/artic le/view/166
To Browse

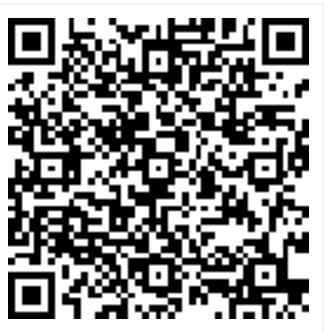

Manuscript Received 06-04-2020

Conflict of Interest No
Review Round 1 16-04-2020

Funding

$\mathrm{Nil}$

Review Round 2
22-04-2020
Ethical Approval
Yes

Review Round 2

Yes
Review Round 3

Plagiarism X-checker $16 \%$
Accepted 28-04-2020

(c) 2020 by Mohan B Choudhary, Ganesan G Ram and Published by Siddharth Health Research and Social Welfare Society. This is an Open Access article licensed under a Creative Commons Attribution 4.0 International License https://creativecommons.org/licenses/by/4.0/ unported [CC BY 4.0]. 


\section{Introduction}

Fractures of the neck of the femur are commonly seen in the geriatric population resulting in significant morbidity and mortality [1]. Communication at the posteromedial cortex, postero-lateral communication, and fractures extending into the proximal femoral diaphysis result in the unstable neck of femur fractures which are often challenging to treat. The treatment of such fractures must aim at surgical correction while achieving early mobilization. Primary arthroplasty helps in rapid mobilization while decreasing the chances of implant failure and complications such as deep vein thrombosis, pulmonary complications, infection, and bedsores. The purpose of this can be fulfilled either by Bipolar Hemiarthroplasty as well as by Total Hip arthroplasty. The aim of this study is to determine whether total hip replacement is clinically and functionally better than bipolar hemiarthroplasty in the neck of femur fracture in older patients.

\section{Materials and Method}

Study settings: It is a study done at Sri Ramachandra Medical College, Chennai, and Velammal Medical College, Madurai on 70 patients diagnosed with the unstable neck of femur fractures who were treated with bipolar hemiarthroplasty and total hip replacement.

Duration and type of study: The study period was from April 2018 to September 2019. This is a prospective control study. Since it's a period study sample size was not calculated and all the patients who underwent the procedure during that period and who was willing for the study became the sample. Institutional ethics committee clearance was obtained

Inclusion criteria: The inclusion criteria were aged more than 60 years and Unstable neck of femur fractures as per the Garden classification [2].

Exclusion criteria: The exclusion criteria were pathological fractures, associated fracture of lower limbs, and non-ambulatory patients prior to the injury.

Data collection procedure: The patients who underwent total hip replacement were chosen and categorized as Group 1 (35 cases) and those patients who underwent bipolar hemiarthroplasty were categorized as Group 2 (35 cases).
Sampling Method: The decision to do whether the bipolar or total hip replacement was done by card method.

Surgical Procedure: The general condition of the patients was assessed at the time of admission. A thorough clinical and radiological evaluation was done. Any pre-existing co-morbidities were taken into account. The injured limb was immobilized using skin traction till the day of surgery. Patients received either mechanical or chemical thromboprophylaxis following injury. Patients were taken up for surgery after obtaining medical fitness. On the day of the procedure, the injured limb that was previously immobilized with skin traction is adequately prepared. Prior to surgical draping, the skin is cleansed thoroughly with three applications of the povidone-iodine solution.

Intravenous antibiotic prophylaxis with $1.5 \mathrm{~g}$ of cefoperazone and sulbactam was administered to all patients half an hour prior to incision. Thromboprophylactic measures were also carried out. Physiotherapy was initiated on the first postoperative day in the form of bed mobilization, chest physiotherapy, and deep breathing exercises. On the second post-operative day, following wound inspection, the drains were removed. The cohort of patients who underwent bipolar hemiarthroplasty was mobilized full weight-bearing with the support of a walker on post-op day three and the patients who underwent THR were mobilized full weight bearing on the second post-op day. Sutures were removed on postoperative day 12 .

Scoring System: After being discharged from the hospital, patients were followed up and reviewed at regular intervals of 6 weeks, 3 months, 6 months, and at the end of one year. Functional assessment was carried out using the Harris Hip Score [3].

\section{Results}

A higher incidence of fracture was found in the 7079 age group in both total hip replacement (71\%) and bipolar hemiarthroplasty $(74 \%)$ groups. The female population $(52 \%)$ was observed to be more than the male population ( $48 \%)$ in this study. The female population was also found to be more prone to fractures in both -THR group (65\%) and bipolar hemiarthroplasty group $(60 \%)$. The mode of injury most commonly reported in this study was accidental slip and fall with $86.7 \%$ of patients in the THR group and $86.7 \%$ in the bipolar hemiarthroplasty group. 
The comorbidities associated were found to be higher overall in the group that underwent bipolar hemiarthroplasty. The mean operative time (in minutes) was observed to be lesser in the bipolar hemiarthroplasty group (109.3) when compared with the patient group that underwent total hip replacement (148.0). The mean operative blood loss $(\mathrm{ml})$ was observed to be significantly lower in the group of patients who underwent bipolar hemiarthroplasty (191.3) in comparison to the total hip replacement group (228.0). The mean amount of blood transfusions was lower in the bipolar hemiarthroplasty group (20.3\%) when compared to the THR group (23.3\%). The mean duration of stay in the hospital is lower in the THR group (6 days) when compared with the bipolar hemiarthroplasty group (8 days).

Table-1: Complication in both groups.

\begin{tabular}{|l|l|l|}
\hline \multicolumn{1}{|c|}{ Complications } & Bipolar & THR \\
\hline Bedsores & 1 & 0 \\
\hline Chronic hip pain & 4 & 0 \\
\hline Respiratory infections & 1 & 0 \\
\hline Urinary tract infection & 3 & 0 \\
\hline Shortening of $>2 \mathrm{~cm}$ & 0 & 0 \\
\hline Deep wound infections & 1 & 1 \\
\hline Implant failure & 1 & 1 \\
\hline Dislocation & 0 & 0 \\
\hline
\end{tabular}

A higher rate of complications was observed in the bipolar group as evident from table 1 . The functional outcome of patients in both groups was analyzed during their follow up at 6 weeks, 3 months, 6 months, and after 1 year using the Harris Hip Score and graphically represented in Figure 1. In the group that underwent a total hip replacement, the mean Harris Hip Score was 70.6, $74.4,77.68$, and 80.95 at the follow-up visits at week 6,3 months, 6 months, and 1 year respectively. In the group that underwent bipolar hemiarthroplasty, the mean Harris Hip Scores were $63.50,66.25,68.72$, and 71.26 at the follow-up visits at week 6,3 months, 6 months and 1 year respectively.

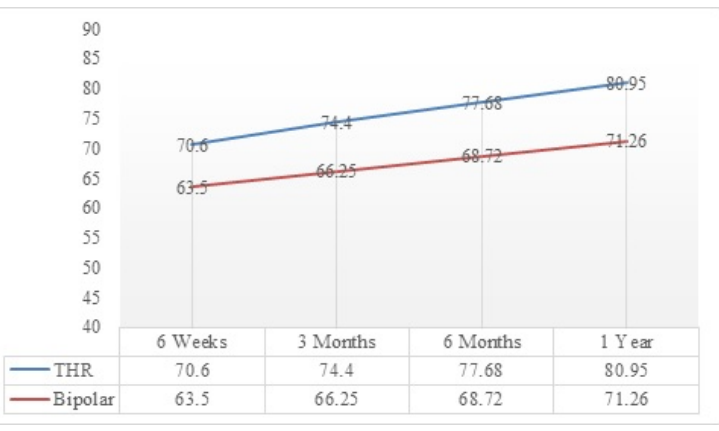

\section{Discussion}

The debilitating consequences of hip fractures include a mortality rate of nearly $30 \%$ in addition to a substantial impairment of the quality of life of the patient. The management of displaced femoral neck fractures is still a subject of debate although a general consensus regarding the operative treatment of undisplaced neck of femur fractures exists [4]. Osteopenia and osteoporosis are highly prevalent in the geriatric population, further influencing the surgical outcome in this patient group [5].

The geriatric population with compromised medical status is particularly susceptible to the stress of multiple surgeries. The age-related weakening of the proximal femur results from a decrease in bone density and loss of tensile and compressive trabeculae. With advancing age, a significant decrease in soft tissue bulk reduces the ability of the muscles and soft tissue to act as shock absorbers, further increasing the likelihood of these debilitating fractures in the elderly. It is thus imperative to choose the most appropriate surgical intervention while also aiming to avoid reoperations.

In agreement with the population standard, in the present study, both THR (65\%) and bipolar hemiarthroplasty groups $(60 \%)$ had a predominantly female population. This could be attributed to the reduced bone mineral density and post-menopausal osteoporosis. The results from Group 1-that underwent THR was found to be superior to the results from Group 2 (bipolar hemiarthroplasty) in terms of better functional outcome, as assessed by the Harris Hip Score and shorter duration of stay in the hospital (6 days versus 8 days). The duration of surgery significantly impacts the treatment outcome in the elderly population. Prolonged surgery often predisposes the patient to higher risks of infection. However it was observed that although THR was associated with a higher operative time of $148 \mathrm{~min}$. when compared to the $109.3 \mathrm{~min}$ of bipolar hemiarthroplasty, complications were reportedly higher in the bipolar group which was in agreement with the findings published by Dawson et al [6]. In the current study, the mean blood loss $(\mathrm{ml})$ was significantly lower in the group that underwent bipolar hemiarthroplasty (191.3) when compared with the group that underwent THR (228.0) corroborating with the observation made by Ossendorf et al [7]. 
The functional outcome of the patient is significantly influenced by the duration of stay in the hospital. The present study found a shorter mean duration of stay in the hospital in Group 1 (6 days) when compared with Group 2 (8 days). The longer duration of hospital stay after bipolar hemiarthroplasty potentially increases the risk of hospital-acquired infections. Yu et al performed a meta-analysis of 12 randomized controlled trials which revealed that THR was associated with a lower re-operation rate with better functional outcomes [8].

Sharma et al concurred that THR produced better functional outcomes and ambulation for elderly patients. Hemiarthroplasty, however, has been reported to have a higher incidence of complications such as acetabular erosion and conversion to THR, although it has the advantage of shorter operation time and less blood loss [9]. The higher mean Harris Hip Score in the THR group reflected the better functional outcome when compared with the bipolar group which was in agreement with the results obtained from the current study.

Wound infection has been reported to be the most frequent complication with hemiarthroplasty and the most frequent complication with after total hip arthroplasty [10]. In the present study, one case each of deep wound infection was observed in both groups. This could be attributed to the influence of post-traumatic osteoarthritis, female gender, and age. Debridement was carried out and IV antibiotics were administered. The infection resolved after 3 weeks of treatment. In the present study only one case, each of implant failure was identified in both groups which were in agreement with Bekerom et al and Dawson et al [11]. However, the rate of revision in older patients undergoing THR could be lower due to the reduced functional demand as well as a lack of adequate fitness to undergo revision surgery. Other complications encountered in the current study included bedsores, UTI, lower respiratory tract infections, and chronic hip pain.

Grade 1-bed sores over the gluteal region were observed in one patient (3.3\%) in Group 2 whereas none of the patients $(0 \%)$ who underwent THR developed bed sores. The bed sores following bipolar hemiarthroplasty could be attributed to the generalized weakness and hence poor compliance with postoperative mobilization. The rapid return to full weight-bearing walking and functional independence with THR thus helps avoid the complication of developing bedsores. Total hip
Arthroplasty, when compared with bipolar hemiarthroplasty, is superior with better functional outcomes, lower re-operation rates, and significant improvement in the quality of life [12]. Although patient-reported satisfaction approaches $90 \%$ after THR, some patients continue to experience chronic pain after elective surgery. In our one-year followup study, 4 patients who had undergone bipolar hemiarthroplasty developed chronic hip pain. This could be potentially be attributed to articular cartilage degeneration in the acetabulum, infection, or loosening of the prosthesis.

\section{Limitations}

The small sample size and one year follow up are the main limitations of the study. However those limitations can not be considered as the outcome changer as the study mainly focusses on the elderly population.

\section{Conclusion}

Total hip arthroplasty is recommended over hemiarthroplasty for the displaced neck of femur fractures in elderly patients owing to the higher Harris Hip Scores and lower re-operation rates.

\section{What does this study add to existing knowledge?}

All the orthopaedician usually prefer hemiarthroplasty in the elderly but the present study clearly points out Total hip arthroplasty is the best option in displaced femur neck fractures in the elderly.

\section{Author's contribution}

\author{
Dr. B. Mohan Choudhary, Dr. Ganesan G Ram \\ conceptualized the study, prepared the study \\ protocol, conducted the data collection, analysis, \\ and manuscript writing.
}

\section{Reference}

01. Van Embden D, Rhemrev SJ, Genelin F, Meylaerts SA, Roukema GR. The reliability of a simplified Garden classification for intracapsular hip fractures. Orthop Traumatol- Surg Res. 2012;98(4)405-408.

doi:

[Article:https://doi.org/10.1016/j.otsr.2012.02.003]

[Crossref] 
02. Ram GG, Govardhan P. In-Hospital Mortality following Proximal Femur Fractures in Elderly Population. Surg J. 2019;5(02)e53-e56.

doi: [Article:https://doi.org/10.1055/s-00391692995][Crossref]

03. Ram GG, Rajesekaran R, Vignesh Jayabalan DP. Is Functional outcome of Cementless Total Hip Replacement better than cemented one?. Sch Acad J Biosci. 2016;4(2)110-113.

doi:

[Article:https://doi.org/10.36347/SAJB.2019.v07i11. 011][Crossref]

04. Bhandari M, Devereaux PJ, Tornetta III P, Swiontkowski MF, Berry DJ, Haidukewych G, et al. Operative management of displaced femoral neck fractures in elderly patients- an international survey. J Bone Joint Surg. 2005;87(9)2122-30.

doi: [Article:https://doi.org/10.2106/JBJS.E.00535] [Crossref]

05. Im G-I, Shin Y-W, Song Y-J. Potentially unstable intertrochanteric fractures. J Orthop Trauma. 2005;19(1)5-9.

doi: [Article:https://doi.org/10.1097/00005131200501000-00002][Crossref]

06. Ossendorf C, Scheyerer MJ, Wanner GA, Simmen HP, Werner CM. Treatment of femoral neck fractures in elderly patients over 60 years of age-which is the ideal modality of primary joint replacement?. Patient Safety Surg. 2010;4(1)16.

doi: [Article:https://doi.org/10.1186/1754-9493-416][Crossref]

07. Dawson D, Milligan D, Callachand F, Cusick L. Hip Hemi-Arthroplasty vs Total Hip Replacement for Displaced Intra-Capsular Hip FracturesRetrospective Age and Sex Matched Cohort Study. The Ulster Med J. 2018;87(1)17. [Crossref]
08. Yu L, Wang Y, Chen J. Total hip arthroplasty versus hemiarthroplasty for displaced femoral neck fractures- meta-analysis of randomized trials. Clin Orthop Relat Res. 2012;470(8)22352243.

doi: [Article:https://doi.org/10.1007/s11999-0122293-8][Crossref]

09. Sharma V, Awasthi B, Kumar K, Kohli N, Katoch P. Outcome analysis of hemiarthroplasty vs total hip replacement in displaced femoral neck fractures in the elderly. J Clin Diagnos Res. 2016;10(5)RC11.

doi:

[Article:https://doi.org/10.7860/]CDR/2016/18638.7 877][Crossref]

10. Cordero-Ampuero J, de Dios M. What are the risk factors for infection in hemiarthroplasties and total hip arthroplasties?. Clin Orthop Relat Res. 2010;468(12)3268-3277.

doi: [Article:https://doi.org/10.1007/s11999-0101411-8][Crossref]

11. Ferrata P, Carta S, Fortina M, Scipio D, Riva A, Di Giacinto S. Painful hip arthroplastydefinition. Clin Cases Miner Bone Metab. $2011 ; 8(2) 19-22$.

[Crossref]

12. Van den Bekerom MP, Hilverdink EF, Sierevelt IN, Reuling EM, Schnater JM, Bonke $\mathrm{H}$, et al. A comparison of hemiarthroplasty with total hip replacement for displaced intracapsular fracture of the femoral neck- a randomised controlled multicentre trial in patients aged 70 years and over. J Bone Joint Surg. 2010;92(10)14221428.

doi: [Article:https://doi.org/10.1302/0301620X.92B10.24899][Crossref] 